\title{
Modeling of an Automatic Transmission for the Evaluation of Test Procedures in a Virtual End-of-Line Test Bench
}

\author{
Jan Röper ${ }^{1} \quad$ Jörn Göres ${ }^{1}$ Clemens Gühmann² \\ ${ }^{1}$ Daimler AG, Germany \{jan.roeper, joern.goeres\} @daimler.com \\ ${ }^{2}$ Chair of Electronic Measurement and Diagnostic Technology, Technische Universität Berlin, Germany \\ clemens.guehmannetu-berlin.de
}

\begin{abstract}
End-of-line tests for automatic transmissions are mandatory to ensure quality and safety. The interaction of unit under test, test bench and test automation leads to a high complexity in the development of test automation and test procedures. Validation of test automation and test procedures requires access to the test bench and the unit under test, both of which are only available close to startup of production. Therefore, virtualization of test bench and unit under test can be used to ease the bottleneck.

Virtualization is a common tool in the development of electronic control units for automotive applications using SIL and HIL technologies. The properties of simulation models for a virtual end-of-line test bench differ from those for classical SIL and HIL environments. In this paper, an automatic transmission model suitable for a virtual end-of-line test bench is presented. The required characteristics of the multiple-disk clutch friction model are discussed in detail. Hydraulics are modeled using a Moore machine to enable simulation of the pressure build-up characteristics during shift operation. With the resulting model, the influence of the key parameter of a test procedure actuating an overlapping gearshift is investigated in a virtual test system.

Keywords: automatic transmission, modeling, virtual test bench, HIL, SIL, end-of-line, friction, hydraulics, disk clutch
\end{abstract}

\section{Introduction}

Automatic transmissions (AT) are tested for quality and safety at the end-of-line (EOL). An example for a test regarding quality is the shifting of gears. The testing of the park break is an example for a safety related test. The EOL test system consists of a test bench, the unit under test (UUT) and a test automation. During the EOL test, test bench and UUT are stimulated by the test automation executing test procedures. The results of the tests are compared to limits and documented in a database for statistical evaluation. Based on the test results, the AT is cleared or sent to rework.

When setting up a new test system, validation of the test automation as well as the test procedures is necessary to ensure smooth operation. Test bench and UUT are only available close to startup of production. Therefore, a virtual test bench is used as a substitute. A virtual test bench requires a model of the AT and the test bench. The control functions of the test bench programmable logic controller (PLC) have to be simulated as well. This also applies to the ECU functions used during the EOL test. For the testing of the automation and its test procedures, the simulation has to be connected to the test automation via the communication interfaces used in the real test bench. Figure 1 shows the components of the real test bench. All components except the test automation are simulated in the virtual test bench.

The main application of AT models in literature is the evaluation of system dynamics for controller design (Runde, 1984). The setup described above can be compared to HIL setups used for the evaluation of ECU functions. Isermann describes the history of HIL systems and shows its use for the development of engine control functions (Isermann et al., 1999). HIL simulation is also applied to PLC program testing for manufacturing equipment (Tomaszunas, 1998; Röck, 2007). However, the execution of ECU functions on none-ECU hardware is more characteristic for SIL setups (Chrisofakis et al., 2011). The virtual test bench is a composition of these virtualization techniques. The coupling of ECU and AT models is carried out in a customized third party SIL environment (Brückmann et al., 2009).

The customized SIL environment used for the implementation of the virtual test bench allows the utilization of real communication interfaces and a real-time simulation mode on a none real-time operating system. The test automation is insensitive to minor timing deviations. However, latency and jitter of the test system are affected by the real-time precision. The effects of latency and jitter on the test automation are discussed in (Röper et al., 2014) and have to be considered when working with the virtual test bench. The models of test bench and AT are 


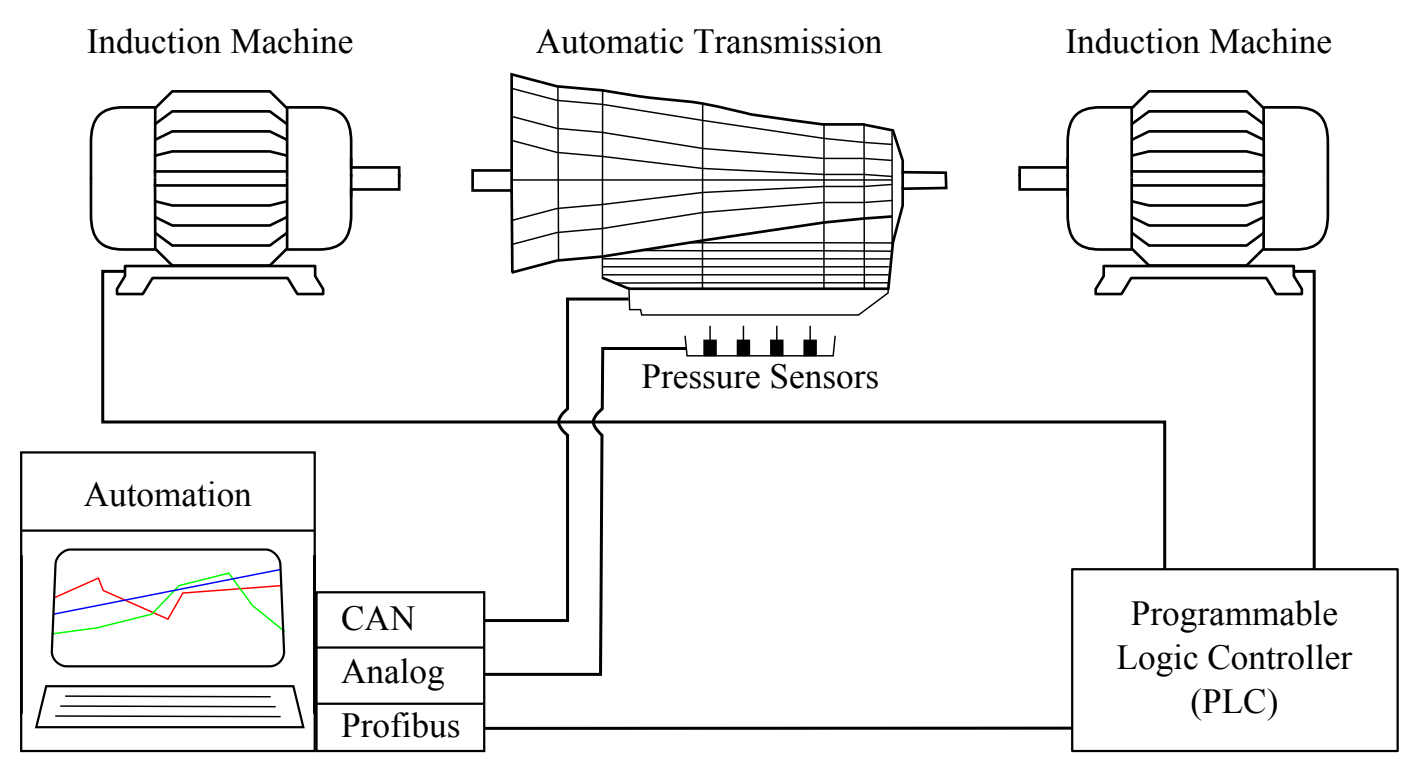

Figure 1. EOL test system

transferred from the Modelica ${ }^{\circledR}$ tool to the SIL as a functional mock-up unit. Simulation is carried out using a fixed step solver.

A similar setup uses a classical HIL as a base (Kuebler et al., 2012). No statements are made about the simulation of ECU functions. In the already publicated HILbased implementation of a virtual test bench, the communication channels are merged to a single Profibus connection neglecting the influence of communication in the real setup.

In this paper, a model of an AT is presented, which fits the specific requirements of the EOL test. When creating a model for the virtual test bench in an early product design stage of the AT, there is a lack of technical data. Parameters which are unknown at this stage are estimated or reused from earlier projects. Such a model enables the test of the automation software as well as the development of test procedures and their pre-parameterization. When transferring test procedures developed in the virtual environment to a real test bench, the parameters have to be verified and possibly readjusted.

The focus in this paper is on the modeling of the characteristics of the pressure build-up in hydraulic clutch actuators as well as on close to zero slip in clutches. The focus results from the tests performed at the EOL: Pressure build-up characteristics have a strong impact on the synchronization during gear shift. After a successful gear shift, the transmission ratio is closely monitored to verify the ability of the clutch to transmit a specified torque. During the EOL test, the induction machine connected to the output shaft is set to speed control, while at the input shaft a constant torque is applied by a second induction machine. The transmission ratio $R_{\mathrm{T}}$ is calculated by:

$$
R_{\mathrm{T}}=\frac{\omega_{\text {in }}}{\omega_{\text {out }}}
$$

with the angular velocity at the input shaft $\omega_{\text {in }}$ and the angular velocity at the output shaft $\omega_{\text {out }}$. A slip in any of the clutches leads to a decrease of the expected speed at the input shaft. In addition, the resulting ratio depends on the speed level which varies during the test. Therefore, the ratio is variable, even if the slip is constant. The following example demonstrates the effect: With an expected ratio of $R_{\mathrm{T}}=1$, a constant overall slip of $\omega_{\text {slip }}=2 \mathrm{rad} / \mathrm{s}$ would lead to a measured ratio $R_{\mathrm{T}}=0.996$ at the set speed $\omega_{\text {out }}=500 \mathrm{rad} / \mathrm{s}$, while the same slip would lead to a ratio $R_{\mathrm{T}}=0.98$ at a set speed of $\omega_{\text {out }}=100 \mathrm{rad} / \mathrm{s}$. The variability of the ratio $R_{\mathrm{T}}$ increases the demand for a model with low slip, since an adjustment of the speed levels may lead to the violation of a limit.

\section{Modeling and Verification}

The virtual test bench model consists of an AT model as well as models of the induction machines and their control. The modeling depth in each submodel is chosen depending on its significance for the EOL test. For example, the oil pumps of the AT are modeled with the states active and inactive, whereas the oil flow, depending on the input speed of the pumps, is not considered. Detailed submodels of the planetary gears, clutches and hydraulic actuators ensure a proper resemblance of the AT characteristics. Below, the detailed submodels of hydraulic actuators and clutch friction are presented. Both hydraulic actuators and clutches are nonlinear systems which require special treatment for real-time simulation. The verification of the test bench model is carried out with regard to its ability to simulate the sticking of closed clutches. 


\subsection{Modeling of Hydraulic Actuator}

Prior to the modeling of pressure build-up in a hydraulic actuator, a detailed analysis of its design is carried out. Figure 2 shows a schematic diagram of a hydraulic actuator. The components of interest are the plunger I, the return spring II and the disks III. In addition, the characteristic pressure curve of the clutch actuator during activation and deactivation is shown as well.

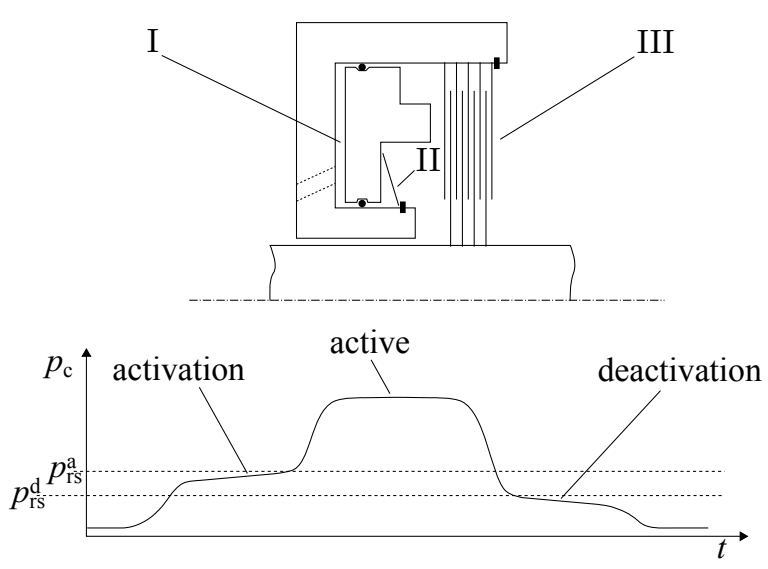

Figure 2. Clutch actuator and pressure characteristics

The clutch is activated by opening a valve that fills the hydraulic cylinder, which leads to the first pressure buildup in the curve. When passing the gap between plunger and disks, the pressure depends on the return spring characteristics. In AT clutches, disk springs are used to provide the return force, which leads to the first plateau at $p_{\mathrm{rs}}^{\mathrm{a}}$ in the curve. Friction counteracts the plunger movement and adds to the return spring force. The second pressure build-up results from the contact force between plunger and clutch disks. The return spring characteristics also dominate the pressure curve when deactivating the clutch. The plateau during deactivation is on a lower pressure level $p_{\mathrm{rs}}^{\mathrm{d}}$, since friction forces act against the spring force when the plunger moves in its starting position. Both of the plateaus feature a small slope. The level of the plateaus as well as the slope is measured during the EOL test to ensure the assembly of the correct return spring type.

A physical model of the assembly presented above would require calculation of contact forces between plunger and clutch disks. This would result in a stiff system and therefore slow simulation due to the necessity of small integrator step sizes (Press et al., 2007). Therefore, an alternative approach is made to implement a real-time capable model. The model includes the electronic control valve which is driven by the ECU. In the model, the target pressure $p_{\text {set }}$ of the actuator is calculated by a lookup table. The pressure build-up is dissected into states which can be represented by the Moore machine shown in figure 3 which is implemented using the Modelica ${ }^{\circledR}$ State Graph library.

The Moore machine gives out different levels of pres- sure. State $S_{1}$ represents the inactive state, where a base pressure $p_{\mathrm{b}}$ ensuring deaeration is provided. State $\mathrm{S}_{2}$ represents the plateau during activation of the clutch. In this state, the pressure $p_{\mathrm{a}}(s)$ is rising with a specified slope. State $\mathrm{S}_{3}$ represents the fully activated actuator where the target pressure $p_{\text {set }}$ is reached, while state $\mathrm{S}_{4}$ is the equivalent to state $S_{2}$ with the pressure $p_{\mathrm{d}}(s)$ when deactivating the clutch.

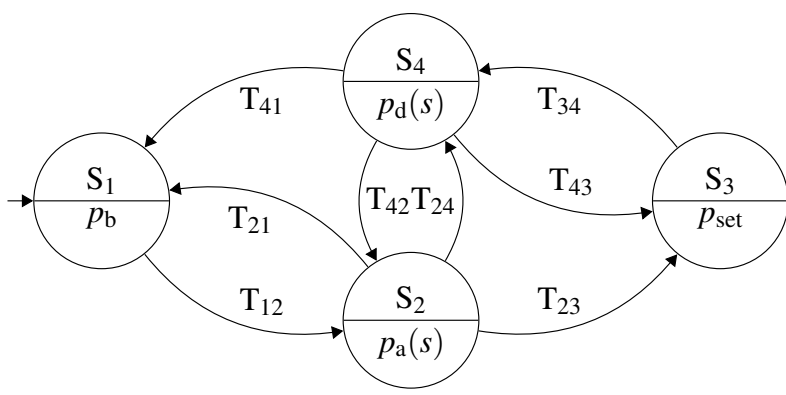

Figure 3. Moore machine of clutch actuator

The transition conditions are summarized by table 1 . Fully activating and deactivating the clutch corresponds to a walkthrough of the states $S_{1}$ to $S_{4}$ in rising order followed by the state $S_{1}$. Switching from state $S_{1}$ to state $\mathrm{S}_{2}$ occurs when a signal is set by the ECU, which is big enough to generate a pressure that moves the plunger. State $S_{2}$ is kept active until the gap is covered. The gap is modeled by a linear differential equation:

$$
s=\frac{1}{t_{\mathrm{gap}}} d t
$$

with position variable $s$ ranging from 0 to 1 . The time needed for covering the gap is provided as a parameter $t_{\text {gap. }}$. The hight of the pressure rise during activation of state $\mathrm{S}_{2}$ is provided by the parameter $\Delta p_{\mathrm{rs}}^{\mathrm{a}}$. State $\mathrm{S}_{3}$ becomes active as soon as the condition $s \geq 1$ is satisfied and stays active until the pressure command $p_{\text {set }}$ drops as specified in table 1 .

Table 1. Transition conditions for Moore machine

\begin{tabular}{ccc}
\hline & $p_{\text {set }}$ & $s$ \\
\hline $\mathrm{T}_{12}$ & $\geq p_{\mathrm{rs}}^{\mathrm{a}}-\Delta p_{\mathrm{rs}}^{\mathrm{a}}$ & \\
$\mathrm{T}_{21}$ & $\leq p_{\mathrm{b}}$ & $\leq 0$ \\
$\mathrm{~T}_{23}$ & $\geq p_{\mathrm{rs}}^{\mathrm{a}}$ & $\geq 1$ \\
$\mathrm{~T}_{24}$ & $<p_{\mathrm{rs}}^{\mathrm{d}}$ & $>0$ \\
$\mathrm{~T}_{34}$ & $<p_{\mathrm{rs}}^{\mathrm{d}}$ & \\
$\mathrm{T}_{41}$ & $<p_{\mathrm{rs}}^{\mathrm{d}}$ & $\leq 0$ \\
$\mathrm{~T}_{42}$ & $\geq p_{\mathrm{rs}}^{\mathrm{a}}$ & $>0$ \\
$\mathrm{~T}_{43}$ & $\geq p_{\mathrm{rs}}^{\mathrm{a}}$ & $\geq 1$ \\
\hline
\end{tabular}

The signal generated by the Moore machine is a succession of straight segments with sharp angles. Both pressure build-ups occur instantaneously, which is not conform to the pressure build-up in the real system. The 
pressure build-up can be modeled by the sum of two parallel PT2 element outputs $y_{\mathrm{PT} 2}^{1}$ and $y_{\mathrm{PT} 2}^{1}$, whose parameters are tuned using measurements from a component test bench or simulation results from a physical model. The first PT2 element representing the pressure build-up before covering the gap is fully active when $s=0$ is satisfied, while the pressure build-up after covering the gap is represented by the second PT2 element when $s=1$ holds. While covering the gap, blend factors $K_{\mathrm{B} 1}$ and $K_{\mathrm{B} 2}$ proportional to $s$ are used to calculate the sum of the PT2 elements:

$$
\begin{aligned}
K_{\mathrm{B} 1} & =s, \\
K_{\mathrm{B} 2} & =1-s, \\
p_{\mathrm{s}} & =y_{\mathrm{PT} 2}^{1} \mathrm{~K}_{\mathrm{B} 1}+\mathrm{y}_{\mathrm{PT} 2}^{2} \mathrm{~K}_{\mathrm{B} 2} .
\end{aligned}
$$

Figure 4 a) shows a measured pressure curve $p_{\mathrm{m}}$ and a simulated pressure curve $p_{\mathrm{s}}$, both scaled by the factor $1 / p_{0}$. The characteristic curves show a good agreement. Oscillations in the measured signal resulting from the pressure control are absent in the simulated signal, since the pressure control is not modeled. At $t=0.4 \mathrm{~s}$ the simulated pressure curve deviates from the measurement. The simplified model is not able to fully represent the physical effects when the contact between plunger and disks is established, which has an influence on the synchronization. The influence of the height of $p_{\text {set }}$ on the pressure characteristics is neglected as well. Figure 4 b) shows the position variable $s$ which rises linear during activation.
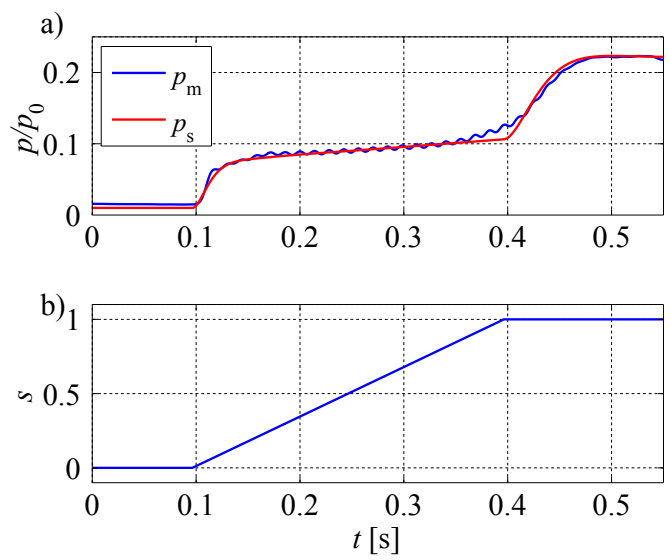

Figure 4. Clutch activation a) pressure and b) position

The utilization of the variable $s$ enables realistic simulation of complex shift operations, for instance pre-fill of the clutch actuator or termination of a shift operation before synchronization. Figure 5 shows pressure, position variable and active state resulting from a normal shift I, an activation of the clutch from a residual pressure II (shift operation starts in state $S_{4}$ instead of $S_{1}$ ) and a prematurely terminated shift operation III. When activating the clutch from a residual pressure, the state
$\mathrm{S}_{2}$ is held for a shorter time, since the position variable $s$ is greater than zero when the pressure command is issued. The same effect occurs when terminating a shift operation. In this case, the pressure drop in state $S_{4}$ is taking less time than in the case of a normal shift operation.
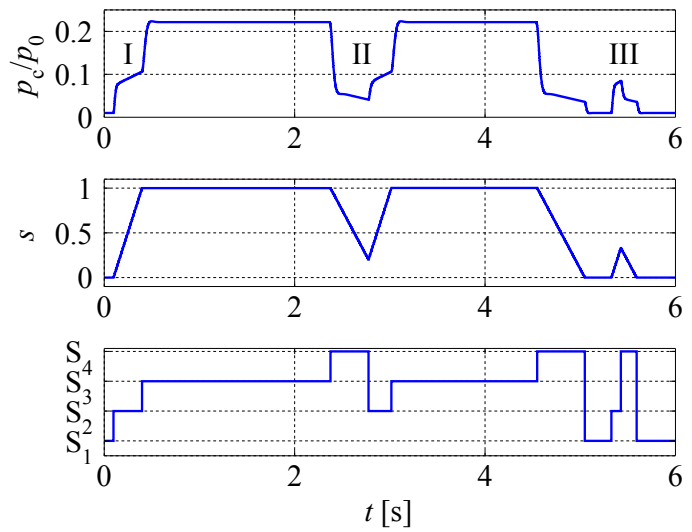

Figure 5. Shift operation scenarios I-III

\subsection{Modeling of Multi Disk Clutch Friction}

The hydraulic actuator model described in the preceding section enables the calculation of the normal force within the clutch. The normal force $F_{\mathrm{N}}$ and the clutch friction coefficient $\mu$ yield the ability of the clutch to transmit torque. The friction coefficient $\mu$ depends on the properties of the friction system, resulting from the tribological system of clutch disks and lubricant, as well as the relative speed $v_{\text {rel }}$ of the shafts. The speed dependency of friction can be considered by expressing the coefficient of friction as a function of $v_{\text {rel }}$ for a specific system. The clutch torque capacity $T_{\text {cap }}$ defines the maximum stick and slip torque, which is determined by:

$$
T_{\text {cap }}=\mathrm{N} \frac{r_{\mathrm{i}}+r_{\mathrm{a}}}{2} \mu\left(v_{\text {rel }}\right) F_{\mathrm{N}},
$$

with the inner disk radius $r_{\mathrm{i}}$ and the outer disk radius $r_{\mathrm{a}}$ as well as the number of disk couples N. Figure 6 shows the typical characteristics of the torque capacity in an AT disk clutch based on a measurement from (Mosbach, 2002). If $v_{\text {rel }}>0$ is true, the transmitted torque $T$ equals the torque capacity $T_{\text {cap }}$. In the case of zero relative velocity, $T$ can take any value between zero and $T_{\text {cap }}$. In this case, $T$ becomes a constraining torque resulting from the torque applied via the shafts. Mathematically, $T$ is a set-valued function for $v_{\text {rel }}=0$ (Lantos and Márton, 2011).

In the following section, the clutch friction system is replaced by a linear contact between a mass and a surface. The considerations made with this simplified system can be transferred to a rotational contact without modifications. 


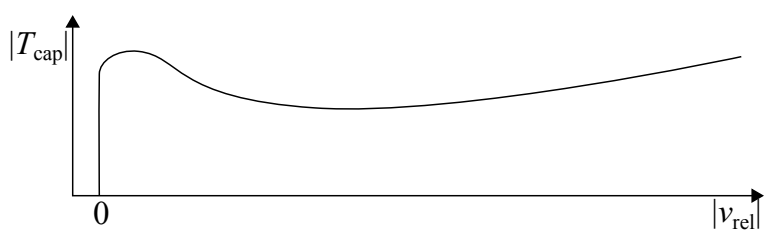

Figure 6. Torque capacity characteristic

There exist numerous friction models to determine the force transmitted when $v_{\text {rel }}=0$. Mare gives a review of different friction models and categorizes them into three types depending on their ability to model contact dynamics (small tangential deformations in the contacting surfaces without sliding) and depending on whether the system dynamics properties are parameters of the friction model (Mare, 2012). The three types are:

1. static and mass free,

\section{2. dynamic and mass free,}

\section{3. mass integrated.}

In static and mass free models the set-valued function at $v_{\text {rel }}=0$ is replaced by a function with a steep rise. Therefore, this type of model can not be used when true sticking with $v_{\text {rel }}=0$ is required. The deviation of $v_{\text {rel }}$ depends on the steepness of the function, which is limited by the computational effort. A steep rise leads to a stiff system which makes small step sizes necessary. An implementation of the type 1 model known as the classic friction model estimates the friction force $F_{\text {fric }}$ with:

$$
F_{\text {fric }}=\tanh \left(v_{\text {rel }} / v_{0}\right) \mu\left(v_{\text {rel }}\right) F_{\mathrm{N}} .
$$

In dynamic and mass free models a differential equation is used instead of the algebraic function in type 1 models. Type 2 models are able to represent true sticking and are often based on physical effects in the contact. Typical examples are the Bristle and the Reset-Integrator model (Haessig and Friedland, 1991). While the Bristle model approximates the contact force by multiple elastic contacts, the Reset-Integrator model uses a single elastic contact which leads to faster computation. The parameterization of type 2 models requires knowledge of the underlying physical effects which is not available when creating models in an early product development stage.

The mass integrated type 3 models employ the external applied force which equals the friction force when sticking. They are simple for a single contact but lead to complex systems when multiple contacts are connected. The implementation of a reusable type 3 friction model for the simulation of an AT is not feasible, since the inertia properties and the structure of the AT are part of the type 3 model. The Karnopp model is the earliest implementation of a type 3 model (Karnopp, 1985). The effort for a multi contact implementation of the Karnopp model is shown by Deur for an AT model (Deur et al.,
2003). An additional submodel for the calculation of the torque at $v_{\text {rel }}=0$ is implemented.

The Modelica ${ }^{\circledR}$ library supplies a clutch model which exploits event handling strategies (Otter et al., 1999). A classification using the three types from above is not possible. Characteristics of the event based friction model are true sticking and mass free. The model sets the relative acceleration $a_{\text {rel }}$ to zero when a change of sign in the velocity is detected. The equation $a_{\text {rel }}=0$ stays active until the force capacity of the contact is exceeded. This model satisfies the demand for small relative velocity in sticking mode, when using a variable step solver with an event searching algorithm. In the case of a sign change in $v_{\text {rel }}$, an event is triggered which stops the integrator. The point of time of the sign change is determined by an algorithm and the integrator is restarted with an adjusted set of equations.

When using a fixed step solver, as required for realtime calculations, the integrator is restarted without searching for the time of the sign change. Therefore, the residual relative velocity in sticking mode depends on the offset between the event and the integrator step after the event. The maximum relative velocity while sticking depends on the step size of the solver and the relative acceleration. The maximum error results from a sign change that takes place right after the integrator evaluated the equations. The time that passes until the event is detected is approximately equal to the integrator step size. The relative velocity resulting from the event based approach is constant once the sticking mode is established, contrary to the relative velocity acquired when using the classical model. Neither the classical nor the event based approach satisfy the requirements stated above.

A friction model that suits the requirements of the virtual test bench is the kpki model (Bai et al., 2013), which uses a structure resembling a limited PI controller. The original implementation does not allow modeling of a peak friction value at zero velocity. Figure 7 shows a novel, extended implementation of the kpki model supporting peak friction. The approach is based on an altered version of the limited PI controller provided by the Modelica ${ }^{\circledR}$ library.

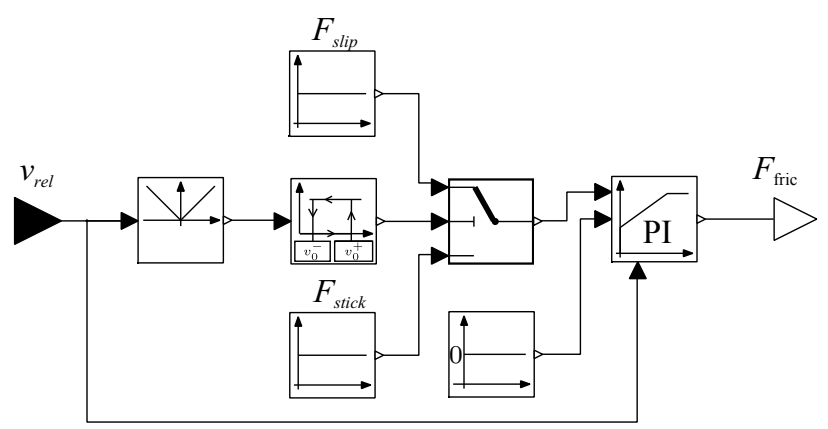

Figure 7. Extended kpki model

The PI controller is based on the work presented in 
(Aström and Hägglund, 1995), where the limit in the controller is set as a parameter. Extending the model to the application of variable limits enables the support of peak friction at zero velocity. The limit is adjusted depending on the relative speed. Since the controller only reacts if $v_{\text {rel }}=0$ is violated, a range representing zero velocity has to be defined. This range can be compared to the approach in the Karnopp model, where the parameter $D V$ is used for this purpose. The model shown in figure 7 contains a hysteresis element defining two speed levels $v_{0}^{+}$and $v_{0}^{-}$for the adjustment of the controller limit. This is necessary for a clean switchover to the stuck mode. If the relative velocity decreases and the switch to the peak friction level is made to early, the controller sets a high friction force to reach zero slip. Since the friction force in the physical system results from the external force, a rising friction force just before reaching zero speed is implausible. Figure 8 shows the curve of the simplified force characteristics implemented with a hysteresis element.

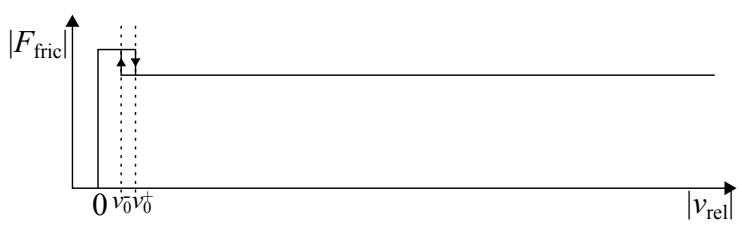

Figure 8. Friction force levels

A standard setup for the evaluation of friction models is shown in figure 9 (Haessig and Friedland, 1991). It consists of a mass with a friction contact to a surface, which is connected to a spring. The system is stimulated by applying a constant speed to the free end of the spring.

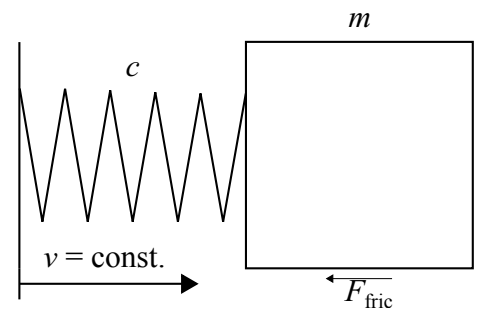

Figure 9. Friction experiment

The compression of the spring leads to a force $F_{\mathrm{c}}$ on the mass. The spring force rises until the peak value of the friction characteristic curve is exceeded $\left(F_{\text {stick }}=\right.$ $0.25 \mathrm{~N})$, resulting in an acceleration of the mass against the remaining friction force $\left(F_{\text {slip }}=0.2 \mathrm{~N}\right)$. The movement of the mass relieves the spring until the damping of the friction brings it to a stop. The process is repeated in the same fashion as long as the spring end is moved. Figure $10 \mathrm{a}$ ) shows the resulting spring and contact force for the extended kpki model and figure $10 \mathrm{~b}$ ) for the classical model. Both models are supplied with a friction characteristic with a peak at zero slip. The classical model does not have the ability to differ between rising and falling speed, which leads to the peaks at the end of the sliding phase. The maximum relative speed during sticking is $5 e-4 \mathrm{~m} / \mathrm{s}$ for the classical model, while the extended kpki model shows a maximum relative speed of $6.7 e-7 \mathrm{~m} / \mathrm{s}$.
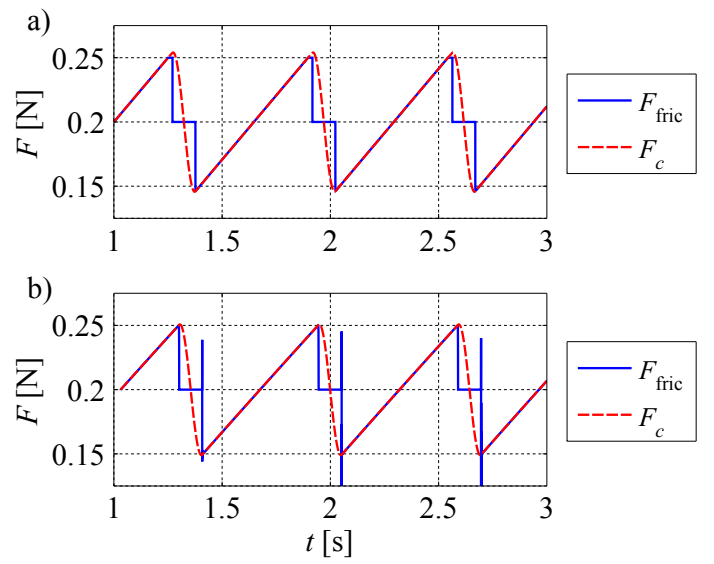

Figure 10. Friction experiment result a) kpki model b) classical model

The parameters of the model include gain $K_{\mathrm{PI}}$ and time constant $T_{\mathrm{i}}$ of the PI controller, as well as the velocities $v_{0}^{+}$and $v_{0}^{-}$that define the switch points of the hysteresis element. When tuning the parameters of the extended kpki model, overshooting and oscillation of the friction force set by the PI controller have to be avoided. Therefore, an analysis of the poles of the system is carried out to fix the PI controller parameters. The time constant $T_{\mathrm{i}}$ is fixed at $T_{\mathrm{i}}=20 \mathrm{~h}$ with the integrator step size $h=0.0001 \mathrm{~s}$ for the explicit Euler algorithm. The resulting numerical stability can be evaluated after the poles are set (Cellier and Kofman, 2006). The system boundary for the linear analysis includes the mass and the friction contact. Figure 11 shows the block diagram representing the model with the external force input $U$ and the relative velocity output $Y$. The mass is represented by the transfer function $G_{m}(s)=K_{m} \frac{1}{s}$ with $K_{m}=\frac{1}{m}$, while the PI controller is represented by the transfer function $G_{\mathrm{PI}}(s)=K_{\mathrm{PI}}\left(1+\frac{1}{T_{\mathrm{i}} s}\right)$, provided that the limiter is not active.

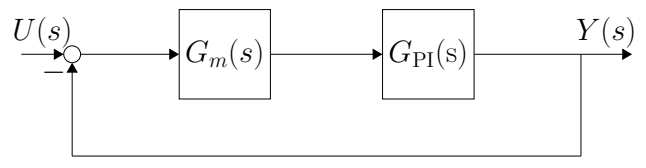

Figure 11. Block diagram

With the block diagram from figure 11 the transfer function of the system can be calculated to:

$$
G(s)=\frac{Y(s)}{U(s)}=\frac{K_{m} K_{\mathrm{PI}} s+K_{m} K_{\mathrm{PI}} \frac{1}{T_{\mathrm{i}}}}{s^{2}+K_{m} K_{\mathrm{PI}} s+K_{m} K_{\mathrm{PI}} \frac{1}{T_{\mathrm{i}}}} .
$$

A dynamic system is oscillatory if it holds at least two complex conjugate poles. The gain $K_{\mathrm{PI}}$ can be used to 
move the poles to the $\mathrm{x}$-axis of the left half pane of the pole-zero diagram to avoid oscillations. The poles can be calculated from the denominator of the fraction in equation 8 by completing the square:

$$
-\frac{K_{m} K_{\mathrm{PI}}}{2} \pm \sqrt{\left(\frac{K_{m} K_{\mathrm{PI}}}{2}\right)^{2}-K_{m} K_{\mathrm{PI}} \frac{1}{T_{\mathrm{i}}}} .
$$

The poles are located on the $\mathrm{x}$-axis of the left half pane if the square root term is none negative. When setting the parameters $m=0.1 \mathrm{~kg}$ and $c=100 \mathrm{~N} / \mathrm{m}$ (Haessig and Friedland, 1991), a value of $K_{\mathrm{PI}}>200$ leads to a positive square root term and therefore to a dynamic system without oscillation. The best results regarding approximation of true sticking are obtained with $K_{\mathrm{PI}}=600$. The product of the resulting poles and the step width satisfy the stability domain of the explicit Euler algorithm.

After setting the parameters $T_{\mathrm{i}}$ and $K_{\mathrm{PI}}$, the parameter $v_{0}^{+}$can be calibrated by a simple experiment. Subjecting the mass to an external force leads to a relative velocity in the contact which is counteracted by the PI controller. The parameter $v_{0}^{+}$has to be higher than the velocity induced by an external force step to the peak force level from figure 8 . Setting a smaller value of $v_{0}^{+}$triggers the lower friction level intended for the sliding mode. The same applies to an abrupt reduction of the external force to zero during sliding. A parameter $v_{0}^{-}$with the same value as $v_{0}^{+}$would lead to a premature jump to the stick friction level. The effect of a premature jump to the stick friction level is visible in the simulation results from the classical model in figure $10 \mathrm{~b}$ ). Therefore, a low value for $v_{0}^{-}$is desirable. If the value for $v_{0}^{-}$is chosen too small, the controller will never reach the stick mode after sliding. Figure 12 illustrates the performance of the model when stimulated by step signals. The relative speed resulting from a step to the maximum friction force (blue curve) stays within the boundary set by $v_{0}^{+}$, while the relative speed resulting from a step to zero force (red curve) does not trigger the peak friction force after passing $v_{0}^{-}$. Both curves show a discontinuity, which results from the limiting within the PI controller (magnified area).

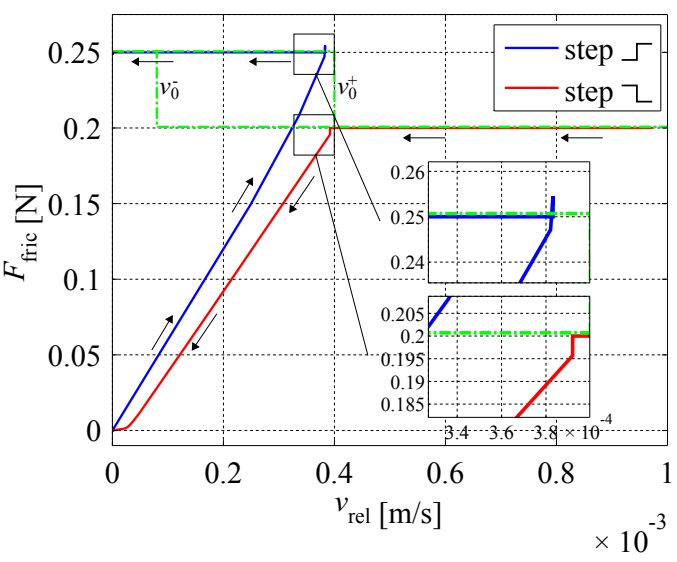

Figure 12. Calibration of $v_{0}^{+}$and $v_{0}^{-}$
The model developed for the linear contact example can be transfered to a clutch model. When setting the parameters in a model with multiple clutches, the closed clutches which are not in the focus of the analysis at the moment can be replaced by ideal, slip-free connections. Modeling of drag torque in the clutch can be achieved by applying a small normal force even if the hydraulic actuator is inactive.

\subsection{Verification}

The suitability of the model for the execution of test procedures with regard to low slip in closed clutches has to be verified as well. The verification is carried out by measuring the transmission ratio while executing a test procedure on the virtual test bench. Table 2 shows the results of the verification and the design values $R_{\mathrm{T}}$ of the transmission ratio presented by (Dörr et al., 2014). The transmission ratio is evaluated in steady state $R_{\mathrm{T} \text {,sim }}^{\text {stat }}$ and dynamic state $R_{\mathrm{T} \text {,sim }}^{\mathrm{dyn}}$. A representative steady state occurs after a successful gear shift. For evaluation of the dynamic state the worst case scenario was chosen: During acoustic measurement the load torque changes its sign, which demands rapid adjustment of the friction force. The transmission ratio is exactly emulated in steady state, while the deviations are small in dynamic state. The biggest deviation from the design value can be observed in the reverse gear with $\Delta R_{\text {sim }}^{\mathrm{dyn}}=0.019$, which is sufficiently small.

Table 2. Verification with test procedure

\begin{tabular}{cccc}
\hline Gang & $R_{\mathrm{T}}$ & $R_{\mathrm{T}, \text { sim }}^{\text {stat }}$ & $R_{\mathrm{T}, \text { sim }}^{\text {dyn }}$ \\
\hline 1 & 5.503 & 5.503 & 5.496 \\
2 & 3.333 & 3.333 & 3.330 \\
3 & 2.315 & 2.315 & 2.311 \\
4 & 1.661 & 1.661 & 1.658 \\
5 & 1.211 & 1.211 & 1.203 \\
6 & 1.000 & 1.000 & 0.996 \\
7 & 0.865 & 0.865 & 0.863 \\
8 & 0.717 & 0.717 & 0.715 \\
9 & 0.601 & 0.601 & 0.600 \\
$\mathrm{R}$ & -4.932 & -4.932 & -4.913 \\
\hline
\end{tabular}

\section{Simulation and Experiment}

With the model presented above, a test procedure executing an overlapping gearshift is developed. The overlapping gearshift consists of a sequence of five steps illustrated by figure 13. After initialization of the test bench and the UUT, the signal for the activation of clutch $\mathrm{C} 2$ is sent to the AT. After an expiration of a sleep timer, clutch $\mathrm{C} 1$ is deactivated. Activating clutch $\mathrm{C} 2$ before deactivating clutch $\mathrm{C} 1$ is necessary due to the pressure build-up 
characteristics discussed above. The choice of the sleep time parameter $\tau$ has a strong influence on the strain induced in the clutches during gearshift. A parameter study of $\tau$ is performed with the virtual test bench using the original automation in a safe environment. Choosing hazardous values for the parameter $\tau$ shows the consequences without damaging hardware. Measurements on a real test bench are carried out to confirm the characteristics identified by the simulation.

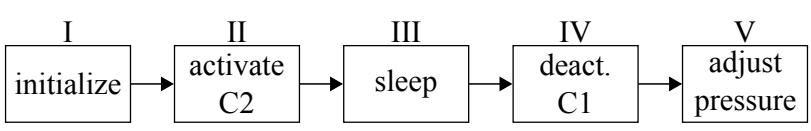

Figure 13. Overlapping gearshift sequence

\subsection{Simulation}

For the parameter study, the parameter $\tau$ is set with values from 150 to $400 \mathrm{~ms}$. Other parameters, like the pressure command for the clutch actuators, are constant during the simulation runs. Figure 14 shows the resulting pressures in the two clutches. The data is synchronized at the beginning of the pressure rise of clutch $\mathrm{C} 2$. The offset in time between the five resulting pressure curves for clutch $\mathrm{C} 1$ is clearly visible. The range chosen for the parameter $\tau$ covers gear shifts with no overlapping, as well as gear shifts with strong overlapping. In the pressure curves of clutch $\mathrm{C} 2$, the pressure adjustment in step $\mathrm{V}$ of the gearshift sequence is visible. The pressure adjustment is carried out with a fixed delay after synchronization of clutch $\mathrm{C} 2$.

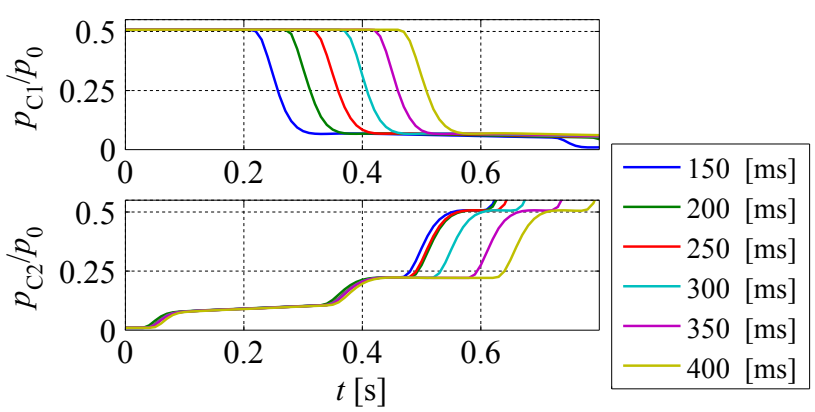

Figure 14. Simulated pressure during gearshift for different values of $\tau$

Figure 15 shows the rotational speed of the input shaft $\omega_{\text {in }}$, as well as the torque $T$ of the input and output shaft. The influence of the sleep time on the synchronization is significant for $\tau \geq 300 \mathrm{~ms}$. In addition, the torque curves change as well. The torque on the input shaft is delayed for greater values of $\tau$, while the characteristics of the torque on the output shaft change dramatically. An additional rise of torque before synchronization of clutch $\mathrm{C} 2$ is visible. For short sleep times $\tau$, the synchronization is triggered by the rising pressure of clutch $\mathrm{C} 2$, while for longer sleep times the synchronization is triggered by the falling pressure of clutch $\mathrm{C} 1$. In the case of longer sleep times, synchronization occurs on a higher pressure level leading to additional, undesirable strain within the transmission.

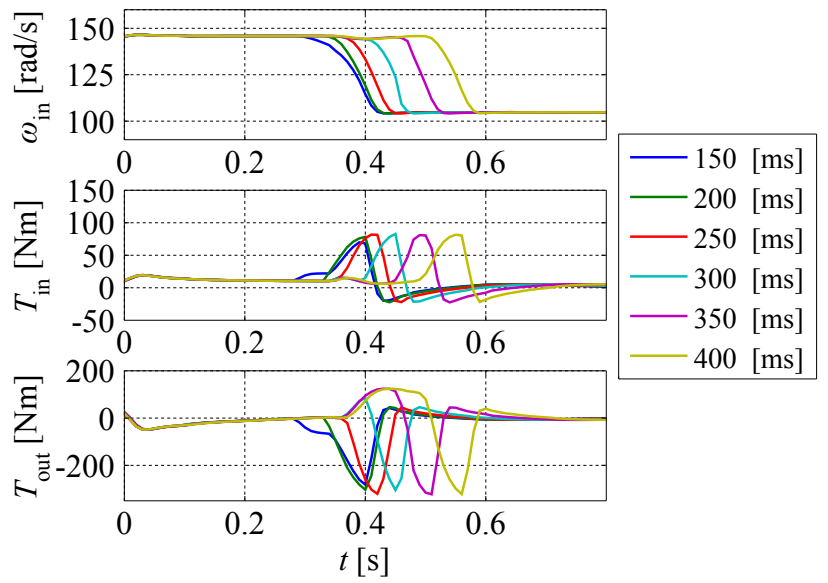

Figure 15. Simulated speed and torque during gearshift for different values of $\tau$

The results from the parameter variation acquired in the virtual environment show that a maximum value of $\tau=250 \mathrm{~ms}$ leads to a clean overlapping gearshift. Longer sleep times lead to additional strain which can possibly damage clutch disks.

\subsection{Experiment}

To verify the simulation results, measurements with the test procedure presented above are carried out on a real test bench. The torque level predicted by the simulation does not exceed the torque tolerated by the test bench. Therefore, the measurements can be performed for all parameter settings used in the simulation. The AT used for the experiments may suffer increased clutch disk wear and is excluded from clearing.

Figure 16 shows the pressure curves measured during gearshift. The influence of the variated sleep time is clearly visible. The time interval between the falling pressures of clutch $\mathrm{C} 1$ is slightly irregular, which is caused by timing deviations in the test system. The signals show low scale oscillations which are not present in the simulation results. This is due to the simplifications in the model omitting pressure supply characteristics and clutch piston friction. The slope of the falling pressure in clutch $\mathrm{C} 1$ differs from the simulated clutch pressure slope, which can affect the time needed for synchronization.

Figure 17 shows the resulting curves for speed and torque. The synchronization of the measured input shaft speed $\omega_{\text {in }}$ is slower than the simulation result and shows an additional undershoot. The undershoot after synchronization is compensated in the simulation by the ideal speed control. The influence of the sleep time on the input shaft speed is significant for $\tau \geq 350 \mathrm{~ms}$, 


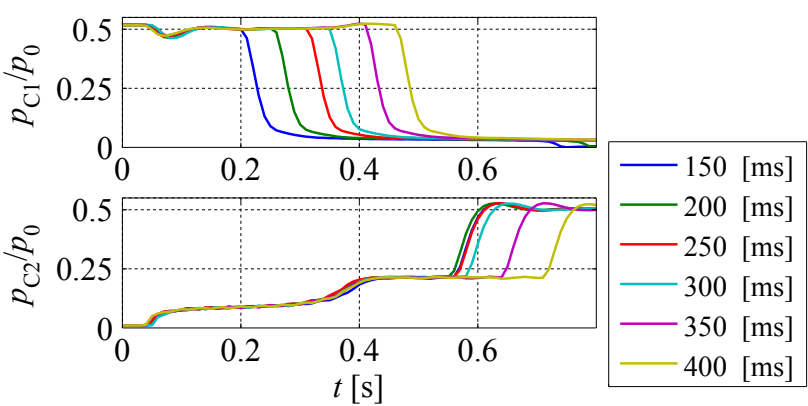

Figure 16. Measured pressure during gearshift for different values of $\tau$

while the torque measurements are already affected for $\tau \geq 300 \mathrm{~ms}$. The maximum of the torque signal at the input shaft $T_{\text {in }}$ shows a disagreement to the simulation. The inertias in the test bench model are only estimates, which leads to the deviation of the input shaft torque $T_{\mathrm{in}}$. Characteristics of the measured and the predicted torque as well as the influence of the parameter $\tau$ are similar. The output shaft torque $T_{\text {out }}$ shows a good resemblance, although the measured signal is smoother, which fits to the elongated synchronization.

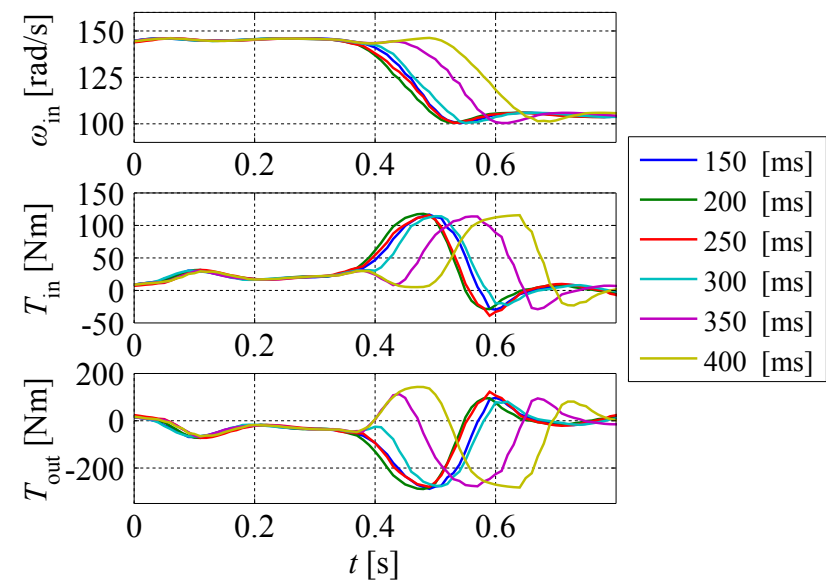

Figure 17. Measured speed and torque during gearshift for different values of $\tau$

Altogether, the simulation shows a sufficient resemblance of the measured signals. The ability to estimate parameters for test procedures is confirmed. In the example shown above, a choice of the parameter $\tau=250 \mathrm{~ms}$ in the virtual environment is valid for the real test bench as well. This holds even though strong simplifications were made in the process of modeling.

\section{Summary and Outlook}

In this paper, an approach for the modeling of an AT in an early stage of product development is presented. In addition, real-time requirements resulting from the integration in a virtual test bench are met. Modeling of a hydraulic clutch actuator as well as modeling of friction is discussed in detail. An extended implementation of the kpki model is applied to a synthetic example of a dynamic system and a method for the parameterization of the friction model is presented as well.

With the resulting virtual test system, the influence of the key parameter for an overlapping gearshift is investigated. The qualitative agreement of the results is confirmed by measurements on a real test bench. Possible improvements for a better quantitative agreement of the results obtained with the virtual test bench include:

- parameterization with speed dependent friction coefficient and exact test bench inertias,

- detailed modeling of controller properties of the induction machine,

- modeling of the effect of the valve target value in the hydraulic actuator.

\section{References}

K. Aström and T. Hägglund. PID controllers: theory, design and tuning. International Society for Measurement and Control Seattle, WA, 1995.

S. Bai, J. Maguire, and H. Peng. Dynamic Analysis and Control System Design of Automatic Transmissions. SAE International, 2013.

H. Brückmann, J. Strenkert, U. Keller, B. Wiesner, and A. Junghanns. Model-based development of a dual-clutch transmission using rapid prototyping and sil. In Getriebe in Fahrzeugen, 2009.

F. Cellier and E. Kofman. Continuous system simulation. Springer, 2006.

E. Chrisofakis, A. Junghanns, C. Kehrer, and A. Rink. Simulation-based development of automotive control software with modelica. In Proceedings 8th Modelica Conference, 2011.

J. Deur, J. Asgari, and D. Hrovat. Modeling of an automotive planetary gear set based on karnopp model for clutch friction. In ASME 2003 International Mechanical Engineering Congress and Exposition, pages 903-910. American Society of Mechanical Engineers, 2003.

C. Dörr, H. Kalczynski, A. Rink, and M. Sommer. NineSpeed Automatic Transmission 9G-Tronic by MercedesBenz. ATZ worldwide eMagazines Edition:, 01:20-25, 2014.

D. Haessig and B. Friedland. On the modeling and simulation of friction. Journal of Dynamic Systems, Measurement, and Control, 113:1256-1261, 1991.

R. Isermann, J. Schaffnit, and S. Sinsel. Hardware-in-the-loop simulation for the design and testing of engine-control systems. Control Engineering Practice, 7:643 - 653, 1999. 
D. Karnopp. Computer simulation of stick-slip friction in mechanical dynamic systems. Journal of dynamic systems, measurement, and control, 107:100-103, 1985.

M. Kuebler, R. Ammann, and M. Wissbach. VIP, der virtuelle Getriebe-Endpruefstand. In 16. VDI Kongress: Berechnung, Simulation und Erprobung im Fahrzeugbau, 2012.

B. Lantos and L. Márton. Nonlinear Control of Vehicles and Robots. Springer-Verlag London, 2011.

J. Mare. Friction modelling and simulation at system level: a practical view for the designer. Proceedings of the Institution of Mechanical Engineers, Part I: Journal of Systems and Control Engineering, 226:728-741, 2012.

C. Mosbach. Das Reibungs- und Reibschwingverhalten nasslaufender Lamellenkupplungen. $\mathrm{PhD}$ thesis, Technische Universität München, 2002.

M. Otter, H. Elmqvist, and S. Mattsson. Hybrid modeling in modelica based on the synchronous data flow principle. In Computer Aided Control System Design, 1999. Proceedings of the 1999 IEEE International Symposium on, 1999.

W. Press, B. Flannery, S. Teukolsky, W. Vetterling, and T. Gould. Numerical recipes, the art of scientific computing. Cambridge University Press, 2007.

S. Röck. Echtzeitsimulation von Produktionsanlagen mit realen Steuerungselementen. $\mathrm{PhD}$ thesis, Universität Stuttgart, 2007.

J. Röper, J. Göres, and C. Gühmann. Analysis of timing and jitter in real and virtual test bench for automatic transmissions. In Simulation and Testing for Automotive Electronics $V, 2014$.

J. Runde. Modelling and Control of an Automatic Transmission. PhD thesis, Purdue University, 1984.

J. Tomaszunas. Komponentenbasierte Maschinenmodellierung zur Echtzeit-Simulation für den Steuerungstest. PhD thesis, Techn. Univ. München, 1998. 